\title{
La influencia del contenido de la historia en el recuerdo de los niños
}

\section{Alvaro Marchesi}

\section{Universidad de Salamanca}

Los dos experimentos anteriores han confirmado la importancia del esquema de la historia para su comprensión y recuperación y han puesto de manifiesto los cambios evolutivos que se producen en su utilización cuando enfrentamos al niño con estructuras narrativas más complejas que se apartan del orden lógico-temporal habitual.

Sin embargo, algunos estudios recientes han insistido en el papel que ocupa también en el recuerdo de las historias el contenido de las mismas. Cuando el contenido es poco familiar al niño, éste pierde parte de la información transmitida al no ser capaz de asimilarla a través de su-conocimiento existente. Pero esta mayor dificultad tiene un efecto importante en la organización que el niño realiza sobre el texto presentado, ya que si el contenido no es familiar, el niño no es capaz de reálizar las inferencias causales y temporales que relacionan los acontecimientos que se producen en la historia. Lo cual debe traducirse no sólo en un nivel de recuerdo inferior, sino también en una "regresión» a estrategias de recuperación más infantiles, que no se utilizarian cuando las causas y las consecuencias expresadas en la narración fueran más familiares.

Para comprobar la influencia de la familiaridad con el contenido en el recuerdo de las historias realizamos un nuevo experimento dentro de las mismas coordenadas teóricas y metodológicas que los anteriores. Construimos dos nuevas historias que se ajustaran perfectamente a la gramática propuesta por Stein y Glenn (1979), cuyo contenido -en cada una de las categorias - fue considerado más difícil que los cuentos utilizados en el experimento anterior. Las historias fueron contadas solamente a niños de seis años, ya que en edades superiores la diferencia entre lo fácil y difícil era mucho menor como demostró un estudio piloto previo. La elaboración de cuentos de contenido menos habitual para los niños de once años los hubiera hecho excesivamente complicados para los más pequeños, por lo que se eligió una sola edad - seis años- que permitiria, además, comparar sus resultados con los obtenidos en esta misma edad en los dos primeros experimentos.

Las historias fáciles y dificiles fueron presentadas de dos formas: ordenadas y mezcladas, de acuerdo con los mismos criterios que se utilizaron en el experimento anterior.

\section{METODO}

\section{Sujetos}

Los sujetos que participaron en este estudio fueron 24 niños de seis años (edad media, 6,8 años) que estudiaban en el Colegio Nacional de Salamanca, al que ya se hizo referencia anteriormente. $\mathrm{El}$ número de niños y niñas fue, aproximadamente, el mismo.

\section{Materiales}

Se construyeron cuatro historias, dos de contenido familiar y otras dos de contenido menos familiar, todas ellas elaboradas según la gramática de Stein y Glenn (1979). Cada categoría estaba formada por cuatro proposiciones.

Las historias de contenido familiar eran las mismas utilizadas en el experimento anterior (cfr. apéndice). Las historias de contenido menos familiar (cfr. apéndice) fueron analizadas por cinco adultos, quienes consideraron que el contenido de todas y cada una de las categorías era más difícil que las proposiciones de las categorías correspondientes en las historias sencillas. Hay que precisar que, mientras que las diferencias entre los sucesos, respuestas internas, ejecuciones, consecuencias y reacciones se consideraron semejantes, no sucedió lo mismo con la categoria introducción, en donde las diferencias eran más acusadas.

En efecto, la dificultad principal de las categorías que forman el episodio se encontraba en la situación elegida en donde se producía el problema, y no tanto en los hechos concretos y acciones realizadas por los personajes, que eran muy semejantes a los de las historias más sencillas.

Las cuatro historias fueron organizadas de dos modos diferentes: ordenadas de acuerdo con el orden prototípico de la gramática, y mezcladas, alterando principalmente la posición de las categorías introducción y consecuencia, siendo su orden el siguiente: suceso, consecuencia, respuesta interna, ejecución, reacción e introducción.

\section{Diseño}

Se dividieron los 24 sujetos en dos grupos iguales que fueron asignados a una de las dos 
condiciones de familiaridad con el contenido: fácil y difícil. Se comprobó el recuerdo de cada uno contándoles dos historias diferentes: ordenada y mezclada, realizándose el oportuno contrabalanceo para evitar el efecto del orden de presentación. A todos los niños se les pidió que recordaran las historias en dos momentos: inmediatamente después de escucharlas y una semana después.

$\mathrm{El}$ diseño resultante fue un diseño factorial mixto $2 \times 2 \times 2 \times 6$, con dos contenidos (familiar y menos familiar), dos tipos de estructura (ordenada y mezclada), dos momentos de recuerdo (inmediato y después de una semana) y seis categorías que constituian cada narración. El único factor intersujetos era la familiaridad con el contenido, siendo los otros tres factores intrasujetos.

\section{Procedimiento}

El procedimiento era idéntico al del experimento anterior, con la única diferencia de que ahora sólo se contaba a cada niño dos cuentos en vez de tres.

\section{Puntuación}

Los análisis realizados en este experimento se centraron en los mismos aspectos que en el experimento anterior y utilizaron técnicas idénticas: número de proposiciones correctamente recordadas en el total del cuento y por categorías, siendo la coincidencia entre evaluadores del 95 por 100 , orden en el recuerdo de las historias con contenido y estructura diferentes, y transformaciones que los niños realizaron a partir del material presentado.

\section{RESULTADOS}

Para el análisis de este experimento se llevaron a cabo siete análisis de varianza. El primer ANOVA contaba con un solo factor intersujetos: la dificultad del contenido (fácil o dificil), y tres factores intrasujetos: el intervalo temporal (recuerdo inmediato y recuerdo diferido), el tipo de estructura (normal y mezclada) y la categoría gramatical (introducción, suceso, respuesta interna, ejecución, consecuencia y reacción). Para cada una de las seis categorías gramaticales se realizaron, por otra parte, otros tantos ANOVAS. En todos los casos, la variable dependiente se midió por el número de proposiciones correctamente recordadas.

A continuación se revisará la influencia de cada uno de los factores.

Los cuentos de contenido familiar para el niño se recuerdan, en general, significativamente mejor $(\mathrm{p}<0,003)$ que los cuentos de contenido no familiar. Pero esto no sucede por igual en todas las categorías, ya que en la respuesta interna, la consecuencia y la reacción, la diferencia no llega a ser significativa.

En cuanto a la estructura, se dan, en general, diferencias significativas entre la estructura ordenada y la estructura mezclada en cuanto a la cantidad de proposiciones recordadas, confirmándose así los resultados obtenidos en el experimento anterior. Tan sólo en una de las categorías, la introducción, se observa una diferencia significativa $(p<0,031)$ en el recuerdo, a favor de la estructura ordenada. Por otra parte, esta variable no interactúa con la dificultad del contenido ni con el intervalo temporal en cuanto al número de proposiciones recordadas.

En cuanto a las categorías gramaticales, agrupando los datos de todas las condiciones se obtiene que la categoría mejor recordada es el suceso, y la peor recordada, la respuesta interna. La variable categoría gramatical presenta una fuerte interacción $(\mathrm{p}<0,001)$ con la variable dificultad del contenido. Esto se traduce en el hecho ya comentado de que tan sólo la mitad de las categorías - la introducción, el suceso y la ejecución- se recuerdan mejor o peor en función del grado de dificultad del contenido, mientras que las otras tres categorías - respuesta interna, consecuencia y reacción- no se ven afectadas significativamente por la familiaridad del contenido. En el caso de la respuesta interna se puede hablar de la intervención del efecto suelo, lo que no puede hacerse con la reacción y la consecuencia, que requieren otro tipo de explicación, que se discutirá en el apartado siguiente.

\section{Mantenimiento del orden de la historia}

Al comparar los niños que han trabajado con narraciones de contenido familiar con aquéllos a los que se ha presentado historias con contenidos menos habituales, se observa que con la estructura ordenada no existen diferencias en cuanto al grado de relación que mantiene el orden de la narración del niño con el orden original (ver cuadro i). En efecto, con ambos tipos de contenido y en ambas sesiones se obtiene una correlación alta con el orden ideal propuesto por la gramática, orden que también corresponde al texto original que se ha proporcionado al niño. No sucede lo mismo cuando se ha utilizado la estructura mezclada. Aunque el recuerdo de las narraciones de contenido no familiar es significativamente peor que el de las narraciones familiares, en cuanto al orden, los cuentos de contenido difícil en la condición mezclada presentan, paradójicamente, índices de correlación más altos que los de contenido fácil (ver cuadro 1). Además, estos índices de correlación son elevados tanto con respecto al orden mezclado que se ha proporcionado al niño como con respecto al orden gramatical ideal. Este hecho que, aparentemente, es contradic- 


\begin{tabular}{|c|c|c|c|}
\hline \multirow[b]{2}{*}{ Contenido } & \multirow{2}{*}{$\begin{array}{c}\text { Ordenada } \\
\text { OR } \times \text { OT }\end{array}$} & \multicolumn{2}{|c|}{ Mezclada } \\
\hline & & OR $\times$ OT & OR $\times$ OGR \\
\hline \multicolumn{4}{|l|}{ I. sesión } \\
\hline 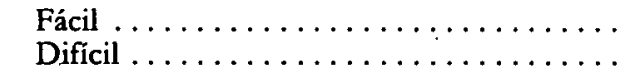 & $\begin{array}{l}0,95 \\
0,98\end{array}$ & $\begin{array}{l}0,36 \\
0,91\end{array}$ & $\begin{array}{l}0,75 \\
0,86\end{array}$ \\
\hline $20^{\circ}$ sesión & & & \\
\hline $\begin{array}{l}\text { Fácil } \ldots \ldots \ldots \ldots \ldots \ldots \ldots \ldots \ldots \ldots \ldots \ldots \\
\text { Difícil } \ldots \ldots \ldots \ldots \ldots \ldots\end{array}$ & $\begin{array}{l}0,99 \\
0,98\end{array}$ & $\begin{array}{l}0,17 \\
0,46\end{array}$ & $\begin{array}{l}0,77 \\
0,95\end{array}$ \\
\hline
\end{tabular}

torio, ya que ambos órdenes no coinciden, se explica porque estos niños, al verse enfrentados a contenidos y estructuras poco familiares, recurren muy frecuentemente a los mecanismos que ya se habian observado en algunos niños de forma aislada cuando el contenido era más familiar. Estos mecanismos consisten en la suspensión de una o varias de las categorías que impiden la coincidencia entre el orden presentado y el orden prototípico. La categoría más olvidada es la introducción, seguida de la ejecución. Una secuencia bastante frecuente es la de suceso - consecuenciareacción que mantiene el orden establecido tanto en la historia mezclada propuesta, como en la gramática original.

Este mecanismo, aunque todavía tiene vigencia, aparece con menos fuerza durante la segunda sesión. Al cabo de una semana, los niños recuerdan las narraciones de estructura y contenido poco familiar, ajustándose más al orden gramatical y alejándose del orden inicialmente propuesto para estas narraciones. Incluso recuerdan introducciones y respuestas internas que no recordaban durante la primera sesión, cuya inclusión en aquel momento hubiera impedido la correspondencia simultánea con el orden gramatical y con la estructura mezclada.

\section{DISCUSION}

El objetivo principal de este experimento era comprobar la influencia del contenido en el recuerdo de las historias. El contenido fue definido en función de la mayor o menor familiaridad que el niño puede tener con el tema presentado, el problema que se suscita y el conjunto de reacciones emocionales y secuencias de acción que el protagonista realiza para resolver la situación planteada. Los resultados confirman la importancia del contenido: los niños recuerdan significativamente mejor las historias cuyo tema es más familiar. Pero este dato adquiere una mayor relevancia si se analiza junto con otros dos: el recuerdo diferencial de las distintas categorias de la historia $y$ de la secuencia del orden en las historias mezcladas en función del nivel de dificultad.

Las importantes diferencias encontradas en la categoría introducción podían ser debidas a la especial complejidad de su contenido en las historias menos familiares. Y la ausencia de diferencias en la respuesta interna se puede explicar porque en ambas historias el número de proposiciones que se recuerda es muy reducido (efecto suelo). Ahora bien, el suceso, la ejecución, la consecuencia y la reacción fueron evaluadas con un grado de dificultad semejante en las historias poco familiares $y$, sin embargo, su recuerdo ha sido desigual. Las proposiciones que formaban el suceso y la ejecución en las historias difíciles se recordaron significativamente peor que en las fáciles, mientras que en la consecuencia y la reacción no se producía ningún tipo de diferencia.

Una posible explicación de este hecho viene apuntada por el lugar que ocupan cada una de estas categorías en la historia. El suceso y la ejecución son categorías del comienzo de la historia, en donde se expresan los origenes y las causas de las acciones posteriores. La consecuencia y la reacción constituyen el desenlace de la historia, la resolución de la misma. Los niños de seis años, enfrentados con un contenido poco habitual, centran su comprensión y recuerdo en el resultado de las acciones, y no son capaces de prestar atención simultáneamente a las causas de esos resultados, a la acción misma, para producir una secuencia coherente y completa. Parece producirse, por tanto, una regresión hacia formas de recuerdo más infantiles. Los niños preescolares manifiestan también un mayor interés por las consecuencias y descuidan los sucesos anteriores que las originan. Los niños de seis años, capaces de organizar su recuerdo en torno a los sucesos y acciones iniciales cuando la historia es sencilla, atienden preferentemente al resultado de las acciones y descuidan sus causas cuando la historia les resulta complicada.

$\mathrm{El}$ recuerdo de las historias mezcladas confirma esta explicación. Los niños en las narraciones difíciles. con estructura mezclada utilizan de forma especialmente rigida el esquema 
prototípico de las historias, lo que les conduce, por un lado, a que el orden de su recuerdo se ajuste extraordinariamente bien al orden ideal (correlación de 0,86 en el recuerdo inmediato y 0,95 en el recuerdo diferido) y, por otro lado, a prescindir de las proposiciones desordenadas, lo que hace posible que la correlación del orden de su recuerdo con el orden del texto sea también muy elevada $(0,91$ en la primera sesión).

\section{Apéndice}

\section{Historia ordenada con contenido menos familiar}

Introducción El carbón es muy necesario para los hombres

) y hay que sacarlo de la tierra.

Para ello se hacen túneles debajo del suelo

Suceso que llegan a lugares muy profundos.

$\begin{array}{ll}\text { Suceso } & \begin{array}{l}\text { Hace unos años, un minero que trabajaba dentro de la mina } \\ \text { oyó una fuerte explosión } \\ \text { y notó que muchas piedras caín } \\ \text { y que tapaban la salida. }\end{array} \\ \text { R. I. } & \begin{array}{l}\text { El minero sintió mucho miedo } \\ \text { y se dio cuenta de que podía quedar enterrado. } \\ \text { Entonces se acordó de los consejos que le habian dado } \\ \text { y decidió intentar salir de la mina. } \\ \text { Permaneció sin moverse un buen rato } \\ \text { hasta que no cayeron más piedras. }\end{array} \\ \text { Consecuención } & \begin{array}{l}\text { Entonces se metió más adentro en la mina } \\ \text { buscando la salida por otro camino. } \\ \text { Después de mucho tiempo encontró un agujero } \\ \text { por donde entraba la luz del sol. }\end{array} \\ \text { Reacción } & \begin{array}{l}\text { Entonces trepó por las paredes } \\ \text { y consiguió salir fuera. }\end{array} \\ \text { Todos los mineros le abrazaron al verle } \\ \text { y le felicitaron por la suerte que habia tenido. } \\ \text { Y todos escribieron una carta a los jefes } \\ \text { pidiendo mayor seguridad en la mina. }\end{array}$

\section{Resumen}

Una afirmación cominmente aceptada en las teorias sobre la comprensión y el recuerdo es que el conocimiento previo favorece el proceso de elaborar una representación de cualquier nueva información. En este experimento se examina la influencia de la familiaridad de los niños con el contenido del cuento.

\section{Summary}

A working assumption in current theories of story comprebension and recall is that prior knowledge is used to facilitate the process of understanding and constructing a representation of any new incoming information. This experiment examine the influence of children's familiarity with the content of the story.

\section{Résumé}

Dans les théories sur la comprébension et mémoire on affirme normalement que la connaissance d'auparavant rend plus facile le processus d'elaboration de la représentation d'une nouvelle information. Dans ce travail on examine l'influence de la familiarité des enfants avec le contenu de l'bistoire. 


\title{
¿Todavía más esquemas?
}

\section{Alvaro Marchesi}

\author{
Universidad de Salamanca
}

El origen de este experimento está en un sugestivo estudio de Stein (1977), en el que abordaba la habilidad de los. niños para comprender la contradicción. Para ello construyó una serie de historias que contenian información discrepante. En una de ellas, por ejemplo, se describía a un zorro que nunca estaba dispuesto a ayudar a otros. Sin embargo, en la segunda parte de la historia; el zorro ve a un oso que estaba muerto de hambre y no podía coger un pez, y le ayuda a pescarlo y cocinarlo. Los planes del zorro y la razón del cambio de su conducta no se incluían en la historia. En una primera tarea se pidió a niños de seis y once años que recordaran la historia lo más exactamente posible. En la segunda tarea se les explicó que parte de la información de la historia no había sido' expuesta, por lo que podían añadir todo lo que considerasen útil para completarla. La mayoria de los niños de seis años olvidaba la descripción inicial del zorro y solamente recordaba las acciones y el resultado de la historia. Por el contrario, los niños mayores incluían en su recuerdo las dos partes incongruentes, realizando inferencias para explicar el cambio de su conducta. En la segunda tarea, estos niños añadían más inferencias para describir por qué el zorro estaba dispuesto a ayudar al oso.

Nuestro objetivo no fue tanto delimitar y explicar los cambios evolutivos que se producen en las estrategias que los niños utilizan para abordar - negando o resolviendo- la información contradictoria, sino más bien intentar comprobar un nuevo tipo de hipótesis: los niños tienden a organizar la información de una historia más firme y consistentemente cuando la consecuencia y reacción final es feliz o satisfactoria. Cuando el final de la historia presentada es negativo, los niños incorporan más fácilmente una información contradictoria cuyo resultado sea positivo, alterándose significativamente su recuerdo posterior en la línea del «final feliz». La comprobación de esta hipótesis abriría una nueva línea de reflexión sobre las características del esquema que el niño utiliza en la comprensión y recuperación de las historias o sobre la incidencia de posibles nuevos esquemas, no sólo en el recuerdo de historias sino tambièn en la organización de otros tipos de secuencias de sucesos.

El experimento fue realizado con niños de once años, edad en la que Stein encontró urra habilidad suficiente para resolver la contradicción entre informaciones discrepantes dentro de la misma historia. La necesidad de una cierta complejidad en la información presentada para poder comprobar la influencia de un texto posterior desaconsejó realizar la investigación con niños más pequeños.

\section{METODO}

\section{Sujetos}

Los sujetos fueron 32 niños de once años del mismo colegio nacional de Salamanca en donde se realizaron los dos experimentos anteriores, elegidos al azar entre aquellos que todavía no habían participado en ninguna de las pruebas.

\section{Materiales}

$\mathrm{Se}$ construyeron dos historias sobre las Naciones Unidas (cfr. Apéndice) siguiendo el orden normal de las principales categorías formuladas en la gramática de Stein y Glenn (1979): introducción, suceso, ejecución, consecuencia y reacción, teniendo todas las categorías el mismo número de proposiciones. Dado su escaso recuerdo no se incluyó la categoría «respuesta interna». En la primera historia (historia positiva) se cuenta la existencia de una guerra entre dos países, las Naciones Unidas deciden que el enfrentamiento se termine, los pueblos en guerra aceptan esta decisión y todos los ciudadanos festejan este hecho y la importancia de las Naciones Unidas. La segunda historia (historia negativa) expone al principio los mismos hechos: una guerra entre dos países. El contenido de las categorías introducción y suceso es el mismo que en la primera historia. Sin embargo, la información transmitida después es la opuesta: las Naciones Unidas no consiguen ponerse de acuerdo, el combate continúa y los pueblos en guerra se entristecen y piensan que las Naciones Unidas son inútiles.

\section{Diseño y procedimiento}

Los 32 niños fueron distribuidos en cuatro grupos: dos grupos control y dos grupos experimentales. A los niños de un grupo control se les contó individualmente la historia positiva y se les pidió que la recordaran inmediatamente y una semana después. $\mathrm{El}$ 
mismo procedimiento se siguió con los niños del segundo grupo control, a quienes se contó en cambio la historia negativa.

A cada uno de los grupos experimentales se le contó o bien la historia positiva o bien la negativa, pidiendo también a los niños que la recordaran nada más escucharla y una semana después. La única diferencia entre los grupos controles y experimentales consistia en que estos últimos escucharon una nueva narración sobre las Naciones Unidas tres días después de la primera sesión experimental, lo que se hizo de la siguiente forma.

La clase a la que pertenecían los niños a quienes se contó la historia positiva (se tuvo el cuidado de que todos fueran de la misma clase y que todos los del otro grupo experimental fueran de otra diferente) fue visitada por un adulto desconocido por ellos. Expuso a todos los niños que estaba realizando un trabajo sobre cómo se resumían noticias de la prensa y les leyó un texto para que después lo resumieran lo mejor posible. El texto en cuestión era la historia negativa sobre las Naciones Unidas. Las primeras proposiciones -iguales en las dos historias - habian sido algo modificadas y resumidas para evitar una relación demasiado clara. El resto de la historia - categorias de ejecución, consecuencia y reacción- era exactamente igual al de la historia negativa.

El mismo procedimiento se realizó con la clase en que estaban los niños que habian escuchado la historia negativa, aunque en este caso el nuevo texto era la historia positiva sobre las Naciones Unidas.

Cuatro días más tarde - es decir, una semana después de la primera presentación y recuerdo de la historia a cada uno de los dos grupos- el primer experimentador volvió a llamar a los niños individualmente y les pidió que recordaran la historia sobre las Naciones Unidas que él les había contado una semana antes. Al final les comentaba que otro niño había tenido problemas en el recuerdo porque otra persona les había contado un texto parecido, y si a él le había pasado algo semejante.

\section{RESULTADOS Y DISCUSION}

El análisis de las grabaciones se centró en dos aspectos principales: I) Número de proposiciones recordadas por los niños de los dos grupos de control, y 2) Número de proposiciones en el segundo recuerdo de los niños de cada grupo experimental que correspondían a la narración primitiva o a la narración contradictoria.

Los resultados (cfr. cuadro I) indican que no existieron diferencias significativas entre el primer y segundo recuerdo de los dos grupos de control y en los dos grupos experimentales, y que el recuerdo de los cuatro grupos fue muy semejante en las dos condiciones.

Dos datos merecen destacarse. El número de proposiciones recordadas en los grupos control no presenta diferencias significativas, lo que indica que el recuerdo de historias semejantes positivas y negativas es prácticamente el mismo siempre que no exista información contradictoria. El segundo dato es la ausencia de diferencias entre el recuerdo inmediato y diferido, lo cual contrasta con los resultados obtenidos en los anteriores experimentos. La explicación puede estar en el número de proposiciones que formaban cada historia. En el experimento anterior el número de proposiciones era 24; en éste is. Al ser una historia más breve, su comprensión y recuerdo puede ser más sencillo y mantenerse durante el intervalo temporal. Sin embargo, esta explicación que puede ser válida en cuentos sencillos no parece adecuada en este caso, ya que la historia de las Naciones Unidas era más compleja y la media del porcentaje de proposiciones recordadas fue sensiblemente menor que en las historias anteriores (40 por 100 frente al 6I por 100). El análisis más detenido de las categorías recordadas permite avanzar una nueva explicación. En el recuerdo de la historia de las' Naciones Unidas, la mayoría de los sujetos recuerdan una proposición de cada una de las categorías en la tarea inmediata. Después de una semana los sujetos utilizan el esquema habitual de recuperación y van urellenando" cada una de las categorías, por lo que su recuerdo es muy semejante. No hay que olvidar que en esta historia no se introdujo la respuesta interna, categoría que tiende a olvidarse con más facilidad.

Pero el resultado más llamativo y sorprendente está en la clase de proposiciones recordadas por los grupos experimentales (cfr. cua-

\section{CUADRO I}

\section{Número de proposiciones recordadas en los grupos control y experimental en dos momentos temporales en el recuerdo de la bistoria sobre las Naciones Unidas}

G. C. $\mathbf{I}$

Inmediatamente $\ldots \ldots \ldots \ldots \ldots \ldots \ldots$

Una semana después $\ldots \ldots \ldots \ldots \ldots$

\section{$4^{8}$}

45
G. C. 2

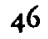

42
G. E. $\mathbf{I}$

is

gI *

G. E. 2

47

$43^{*}$

* Se contabilizaron todas las proposiciones correctamente recordadas en relación con las dos narraciones que habian escuchado.

NOTA.-G. C. 1 = Grupo control 1 (historia positiva); G. C. 2 =Grupo Control 2 (historia negativa); G. E. $1=$ Grupo Experimental 1 (historia positiva); G. E. $2=$ Grupo Experimental 2 (historia negativa). 
dro 2) después de haber escuchado la narracióñ contîradiciuria.

Mientras que los niños que escucharon la historia positiva mantuvieron su recuerdo en torno a esa historia, a pesar de la interferencia del texto negativo, los niños que habían escuchado la historia negativa de las Naciones Unidas se apartaron de esa narración y organizaron su recuerdo la segunda vez con la historia positiva que se les había leído unos dias antes.

Los resultados obtenidos hay que abordarlos con cautela, ya que el número de niños en cada grupo era reducido y no se controló el conocimiento previo que sobre las Naciones Unidas se habian formado estos niños. A pesar de ello, los datos son bastante claros y parecen confirmar, al menos de forma provisional, la hipótesis que se avanzaba al comienzo.
Normalmente los cuentos e historias tienen un resultado satisfactorio. La secuencia lógica se organiza de la siguiente forma: presentación del protagonista, problema con el que se enfrenta, decisión de resolverlo, realización del plan y reacción final que incluye la superación del problema. No es sencillo encontrar un cuento en el que el final sea desastroso para el héroe, ya que el castigo se aplica a los malvados. Los cuentos que nosotros mismos construimos en los primeros experimentos tienen este tipo de contenido, sin que lo hiciéramos de forma deliberada. Las acciones o funciones principales de los personajes de los cuentos tradicionales, según Propp (19281972), indican que una de ellas es el castigo del agresor y la última el matrimonio del héroe y su ascenso al trono. Rodríguez Almodóvar (1982) señala que en los cuentos maravillosos españoles se mantiene el orden y las

\section{CUADro 2}

Tipo de proposiciones recordadas por los dos grupos experimentales en la $2 .^{a}$ sesión

Grupo Experim. I Grupo Experim. 2 \begin{tabular}{l} 
Grupo Experim. I Grupo Experim. 2 \\
Hist. positiva \\
\hline
\end{tabular}

Número de proposiciones de la introducción y el suceso

(iguales en ambos textos) $\ldots \ldots \ldots \ldots \ldots \ldots \ldots \ldots \ldots$

Número de proposiciones del texto original de cada grupo en

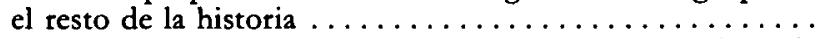

Número de proposiciones del texto contradictorio de cada

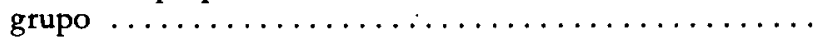

Total

I 2

3 I

8

SI características de estas funciones, aunque desaparezcan algunas de ellas. En ninguno de los cuentos el final se cierra con la derrota del héroe. Todo esto indica que las expectativas que un niño ha adquirido a través de su experiencia sobre el desartollo de los cuentos e historias incluye el éxito final.

Nuestros datos parecen confirmar, desde un planteamiento diferente, la importancia de cómo se resuelve el problema. Los niños que escucharon la historia positiva de la ONU apenas manifestaron ninguna interferencia con la historia negativa escuchada con posterioridad. La información positiva se ajustaba sin dificultad a las historias habituales, por lo que la secuencia de las acciones fue organizada de forma coherente y estable. La nueva información negativa, menos esperada, no llegó a interferir en el recuerdo posterior. La estrategia de recuperación utilizada por el niño, basada no sólo en lo que hemos denominado repetidas veces «esquema de la historia», sino posiblemente también en que los personajes cumplían sus funciones propias y resolvian el problema, les permitió recordar fácilmente la historia original y separar la información contradictoria.
La situación inversa se produce en el grupo de niños que escuchaban en primer lugar la historia negativa, una historia cuyos resultados son inesperados. Su recuerdo después de una semana podria haberse mantenido (es lo que sucede en el grupo control). Pero al haber escuchado una nueva historia, acorde con las narraciones habituales, su recuerdo se aproxima más a esta última al vincular estas proposiciones con la secuencia lógica y con el resultado final satisfactorio que los niños posiblemente utilizaron para recuperar el contenido de la historia. Hay que reconocer que en este grupo las cosas no están tan claras, ya que unos niños recordaron solamente la historia positiva mientras que otros mantuvieron la contradicción y recordaron proposiciones mezcladas de una y otra.

A partir de estas consideraciones podemos avanzar la idea de que el esquema de la historia, entendido como el conjunto de expectativas sobre las unidades encontradas en la historia y su secuencia lógico-temporal, no da cuenta suficiente de todas las expectativas que el niño ha adquirido sobre las historias y que utiliza en su comprensión y recuerdo. Las funciones de los personajes, y especialmente el 
resultado satisfactorio del problema que se ha planteado en la historia - triunfo del protagonista y castigo del agresor--, parecen formar parte también de las expectativas que las personas tienen sobre cuál es el correcto y "lógico" desarrollo y desenlace de una historia. O tal vez este nuevo tipo de expectativas no se produzca solamente en la comprensión de las historias sino que forme parte del conocimiento general que los niños en nuestra cultura adquieren sobre la secuencia de las acciones humanas: los problemas deben ser siempre resueltos, los protagonistas siempre tienen que ganar. Esto podría dar lugar a un amplio tema de debate a nivel educativo, pero nuestro experimento y nuestros datos son demasiado provisionales para intentar plantearlo.

\section{Resumen}

Este experimento fue realizado para examinar la influencia de la información discrepante en el recuerdo de bistorias previamente escucbadas. Los niños fueron más influidos cuando la nueva bistoria tenía un final feliz. Los resultados obtenidos se interpretaron en la linea de que el esquema de la bistoria no debe estar constituido solamente por las categorias propuestas por la gramática de la bistoria.

\section{Summary}

This experiment was conducted to examine the influence of information which is not consistent with the story previously beard. Children were more influenced when the new story bad a happy end. The implications of this finding are discussed in terms of the assumption tbat not only structure grammar is needed to describe children's scbemata to processing stories.

\section{Résumé}

Dans ce travail on a etudie Pinfluence de Pinformation contradictoire sur le souvenir des histoires écoutées auparavant. Les enfants ont été plus influentés quand la nouvelle bistoire avait un final beureux. Les résultats obtenus indiquent lexistence d'autres élements dans le scbeme de Pbistoire, en plus des catégories proposées par la grammaire des bistoires. 